\title{
Diagnostics d'imagerie $X$ par microscopes $X$ à miroirs développés dans le cadre du programme LIL
}

\author{
R. Rosch, J.-Y. Boutin, J.P. Le Breton, G. Lidove et N. Douvry \\ CEA, BP. 12, 91680 Bruyères-le-Châtel, France
}

\begin{abstract}
Résumé : Cet article présente les premiers diagnostics d'imagerie $\mathrm{X}$ à base de microscopes à miroirs mis en cuvre sur la chambre d'expériences de la Ligne d'Intégration Laser (LIL) implantée au CEA-CESTA. Ces diagnostics comprennent trois éléments de base : un bloc optique, qui assure les fonctions d'imagerie et de sélectivité spectrale (dans les domaines $[100 \mathrm{eV}-1 \mathrm{keV}]$ et $[1-5 \mathrm{keV}]$ ), un détecteur rapide (caméra à balayage de fente ou tube obturateur) et un système d'insertion. Ils permettent d'observer des détails inférieurs à $10 \mu \mathrm{m}$ sur un champ de $1 \mathrm{~mm}$ (ou $30 \mu \mathrm{m}$ sur $3 \mathrm{~mm}$ ) au niveau du plasma source. La qualification des microscopes sur un banc $\mathrm{X}$ spécifique du CEA-DIF est également décrite.
\end{abstract}

\section{INTRODUCTION}

La LIL sera mise en service fin 2002, avec un quadruplet du futur Laser Mégajoule (LMJ). Elle délivrera environ $24 \mathrm{~kJ}$ à $0.35 \mu \mathrm{m}$ au centre d'une chambre d'expériences de $4.5 \mathrm{~m}$ de diamètre. Les premières expériences seront consacrées à la caractérisation du procédé de lissage retenu pour le LMJ. Dans une deuxième phase, la LIL disposera de 8 faisceaux élémentaires qui seront utilisés avec un schéma éclaté en deux fagots de 4 faisceaux à $50^{\circ}$ de l'axe d'irradiation.

Les plasmas créés par interaction avec des lasers de ce niveau d'éclairement atteignent des domaines de température de plusieurs millions de degrés et des pressions de plusieurs centaines de Mbars. L'émission radiative de ces plasmas dans le domaine $\mathrm{X}$ est le reflet des processus physiques qui assurent le transfert de l'énergie laser à la matière, et son étude permet de remonter directement aux paramètres fondamentaux du plasma (température, densité, état d'ionisation). Toutefois, les plasmas laser sont le siège de phénomènes souvent indésirables, telles les instabilités, qui demandent une connaissance fine du plasma, sur des dimensions caractéristiques de quelques microns.

Les 12 premiers diagnostics plasmas (DP) développés par le CEA-DIF pour la LIL comprennent 2 diagnostics d'imagerie X par sténopé et 4 diagnostics à base de microscopes à miroirs: les DP 1.05, 1.06, 1.08 et 1.09 illustrés sur la figure 1. Leur résolution spatiale élevée (environ $10 \mu \mathrm{m}$ ) sur un champ millimétrique (voire de quelques millimètres) permet de répondre au besoin. 


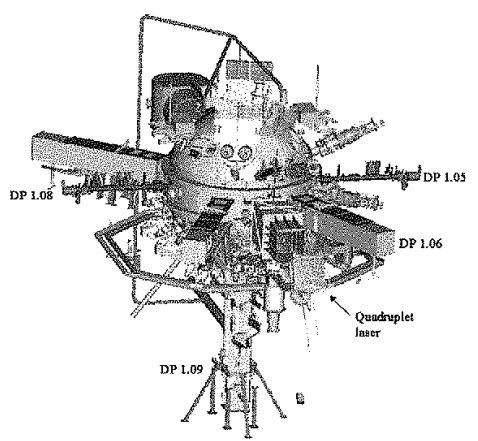

Figure 1. Implantation des 4 premiers diagnostics d'imagerie $\mathrm{X}$ autour de la chambre d'expériences de la LIL.

\section{LES PREMIERS DIAGNOSTICS D'IMAGERIE X DE LA LIL}

Un diagnostic d'imagerie $X$ comprend en général trois éléments : un système de formation d'image (sténopé, ouverture codée, optique à miroirs), un système assurant la sélection spectrale du signal (filtre, miroir multicouche, réseau, cristal) et un capteur (film, caméra à balayage de fente (CBF), caméra à images intégrales (CII)). Les premiers diagnostics d'imagerie $\mathrm{X}$ développés pour la LIL sont de 2 types :

- Imagerie 1D sur caméra à balayage de fente (DP 1.05 et 1.08 ),

- Multi imagerie 2D sur caméra à images intégrales (DP 1.06 et 1.09).

Pour l'imagerie X ID sur caméra à balayage de fente, le concept de mesure utilisé est du type imagerie par couple de miroirs croisés courbes attaqués sous incidence rasante (microscope KB, de type Kirkpatrick et Baez [1]). Le miroir réalisant la résolution dans la grande dimension de la fente de la CBF est dédoublé pour corriger de l'inclinaison de champ (microscope $1 / 2 \mathrm{KBA}$ illustré sur la Figure 2.a) [2]. La reprise d'image est assurée derrière la $\mathrm{CBF}$ par un film visible ou par une $\mathrm{CCD}$. Les diagnostics multi imagerie X $2 \mathrm{D}$ avec résolution temporelle $\left(\mathrm{n}^{\circ} 1.06-1.09\right)$ comprennent plusieurs couples de miroirs de type KB qui permettent de former simultanément plusieurs images des zones émissives du plasma source, dans une direction privilégiée (Figure 2.b). Le nombre de microscopes est multiplié (6 exemplaires) de manière à réaliser le nombre et la distribution spatiale des images souhaitées dans le plan du détecteur. Le détecteur, une caméra à images intégrales (ou tube obturateur), permet de sélectionner temporellement ces différentes images. Pour ces diagnostics, la reprise d'image est également assurée soit par un film, soit par une CCD.

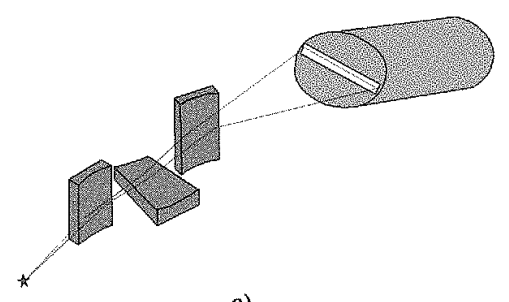

a)

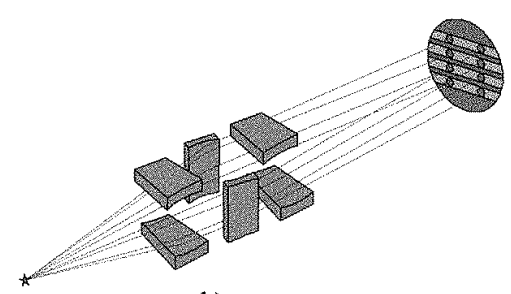

b)

Figure 2. Imagerie par un microscope de type $1 / 2 \mathrm{KBA}$ sur caméra à balayage de fente (a), et par un microscope de type multi KB sur caméra à images intégrales (b).

Les performances de ces diagnostics d'imagerie sont indiquées dans le tableau ci-dessous : 


\begin{tabular}{|l|c|c|c|c|}
\hline & DP 1.05 & DP 1.08 & DP 1.06 & DP 1.09 \\
\hline $\begin{array}{l}\text { Gamme spectrale de } \\
\text { fonctionnement }\end{array}$ & {$[1-5 \mathrm{keV}]$} & {$[100 \mathrm{eV}-1 \mathrm{keV}]$} & {$[1-5 \mathrm{keV}]$} & {$[100 \mathrm{eV}-1 \mathrm{keV}]$} \\
\hline Durée d'analyse & $1,5 \mathrm{et} 25 \mathrm{~ns}$ & $1,5 \mathrm{et} 25 \mathrm{~ns}$ & $<25 \mathrm{~ns}$ & $<25 \mathrm{~ns}$ \\
\hline Résolution temporelle & $30 \mathrm{ps}$ & $30 \mathrm{ps}$ & $80 \mathrm{ps}(4$ instants $)$ & $80 \mathrm{ps}(4$ instants $)$ \\
\hline $\begin{array}{l}\text { Champ d'observation } \\
\text { (2D) }\end{array}$ & $3 \mathrm{~mm} \times 100 \mu \mathrm{m}$ & $3 \mathrm{~mm} \times 100 \mu \mathrm{m}$ & $1 \mathrm{~mm}$ & $1 \mathrm{~mm}$ \\
\hline Résolution spatiale & $30 \mu \mathrm{m}$ & $30 \mu \mathrm{m}$ & $10 \mu \mathrm{m}$ sur $800 \mu \mathrm{m}$ & $30 \mu \mathrm{m}$ sur $2 \mathrm{~mm}$ \\
\hline Nombre d'images & 1 & 1 & $4 \times 2$ & $4 \times 2$ \\
\hline
\end{tabular}

La formule optique des microscopes a été calculée à l'aide d'un logiciel de tracé de rayons adapté au domaine X (logiciel TRACE [3]). La figure 3 donne, comme exemple des possibilités de TRACE, la réponse à une source ponctuelle (dite « réponse percussionnelle ») de microscopes $\mathrm{KB}$ et $1 / 2 \mathrm{KBA}$, dans les directions horizontale et verticale. On remarque l'amélioration du champ dans la direction (courbe en bleu) correspondant au miroir dédoublé. Ces simulations ne prennent pas en compte les défauts (rugosité, erreur de pente).

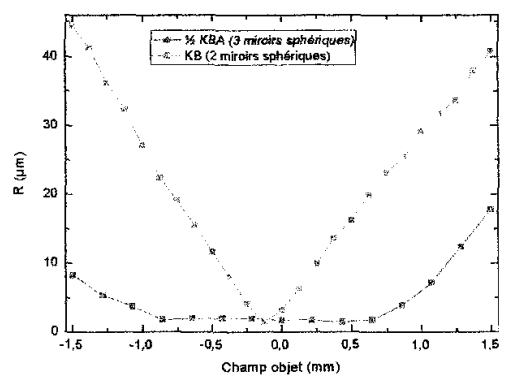

Figure 3. Evolution comparée de la résolution horizontale théorique d'un $1 / 2 \mathrm{KBA}$ (courbe en bleu, avec les miroirs disposés comme sur la Figure 2.a.) et de la résolution horizontale/verticale d'un $\mathrm{KB}$ (courbe en vert).

Les microscopes $\mathrm{X}$ sont équipés d'un système d'alignement intégré destiné à aligner le diagnostic sur le centre de tir. Ce système comporte deux sources lumineuses réglées en laboratoire pour converger vers le centre optique du microscope. Le réglage de ces sources s'effectue en deux étapes : 1/ On réalise des images de grille dans le domaine X pour repérer, par rapport à un motif inscrit sur la grille, le centre de la zone de meilleure résolution du microscope. 2/ On oriente les deux sources vers le centre de cette zone et on les bloque dans cette position. La figure 4 montre le montage réalisé en laboratoire, qui permet de visualiser la grille et les sources lumineuses. Ce banc permet de régler le microscope équipé des sources lumineuses, ce qui sera le cas au démarrage de la LIL, mais également si la fonction « source lumineuse " est assurée par une référence au centre chambre (évolution). On utilise alors un simple miroir sphérique sur le microscope pour renvoyer l'image sur le centre chambre.

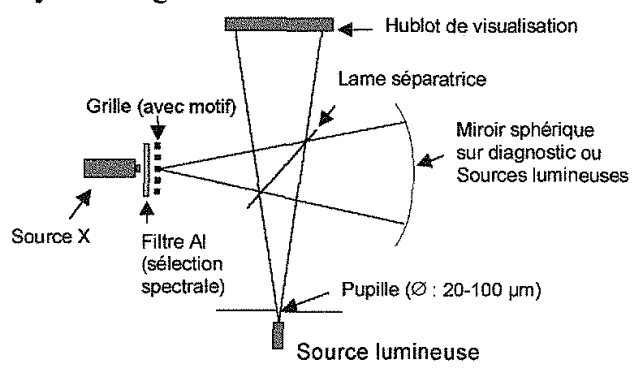

Figure 4. Principe de réglage en laboratoire du système d'alignement intégré au microscope. 


\section{QUALIFICATION DES MICROSCOPES SUR BANC $X$}

La résolution spatiale d'un microscope est mesurée par radiographie $\mathrm{X}$ d'une grille métallique dont on réalise l'image (sur une caméra $\mathrm{CCD}$ ) par le système optique. On utilise pour cela un tube à rayons $\mathrm{X}$ de marque RIBER (source $\mathrm{X}$ dans le domaine [1-8 keV] de quelques millimètres de diamètre). La méthode de mesure «à bord franc » de la résolution utilisée ici s'applique lorsque le pas de la grille est grand devant la résolution à mesurer. Le bord franc du barreau peut alors se représenter par une fonction de Heaviside, dont la dérivée est un pic de Dirac. La largeur de la fonction dérivée donne alors directement la résolution du système. L'image de la grille donnée sur la figure 5 (pas $=340 \mu \mathrm{m}$, trait $=40 \mu \mathrm{m}$ ) a été obtenue dans le domaine [1-1.5 keV] par un microscope de type $\mathrm{KB}$ calculé pour donner une résolution de l'ordre de $10 \mu \mathrm{m}$ sur un champ de $1 \mathrm{~mm}$ (frontale de $500 \mathrm{~mm}$, tirage de $4 \mathrm{~m}$ (grandissement 8), ouverture de collection $0.5 \mathrm{mrad}$ ). Le profil horizontal moyenné sur la zone encadrée en pointillé (pour réduire le bruit de fond) ainsi que la dérivée de ce profil sont représentés sur les Figures 5.b et 5.c. La largeur du profil (à $85 \%$ ) voisine de $9 \mu \mathrm{m}$ est conforme aux spécifications.

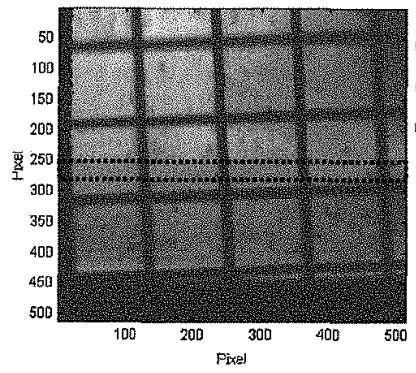

a)

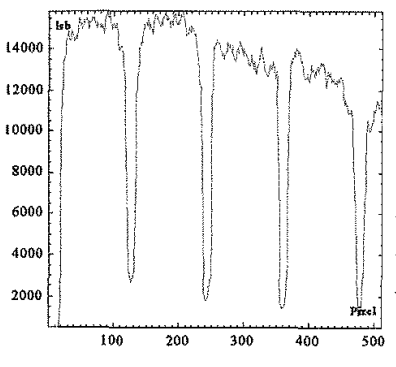

b)

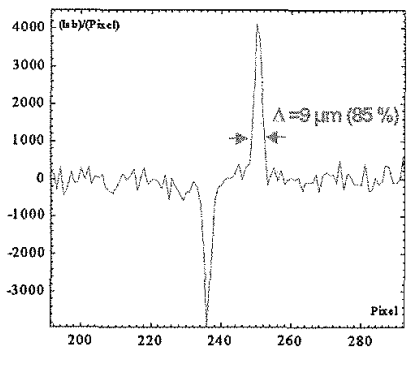

c)

Figure 5. Image d'une grille dans le domaine [1-1.5 keV] (a), profil horizontal (b) moyenné sur la zone encadrée de la figure a) et dérivée du profil horizontal (c, barreau central).

\section{CONCLUSION}

Au démarrage de la LIL, 4 diagnostics d'imagerie à base de microscopes à miroirs seront implantés autour de la chambre d'expériences. Ces diagnostics comprennent un bloc optique assurant une résolution spatiale de 10-30 $\mu \mathrm{m}$ (au niveau de la source) sur un champ important, de 1 à $3 \mathrm{~mm}$. Ils sont couplés à une caméra à balayage de fente (CBF X) ou à un tube obturateur (CII X), avec dans les deux cas une reprise d'image par film visible ou par CCD.

\section{Références}

[1] Kirkpatrick P., Baez A.V., « Formation of Optical Images by X-Rays », J.O.S.A., 38 (9) Sep. 48, pp.766-774.

[2] Sauneuf R. "Large field high resolution $X$-ray microscope for studying laser plasmas », 3412 Rev. Sci. Instrum. 68 (9) Sep 97

[3] TRACE : T. Moreno (société CAMINOTEC) 2001. 